\title{
Predictability of noise-controlled dynamics
}

\author{
G.D. Lythe ${ }^{\mathrm{a}, *}$, M.R.E. Proctor ${ }^{\mathrm{b}}$ \\ ${ }^{a}$ Center for Nonlinear Studies, Los Alamos National Laboratory, B258, NM 87545, Los Alamos, USA \\ ${ }^{\mathrm{b}}$ Department of Applied Mathematics and Theoretical Physics, University of Cambridge, Cambridge, CB3 9EW, UK
}

\begin{abstract}
Noise-controlled dynamics runs counter to usual intuition: the larger the noise the more regular the solutions. We present numerical and analytical results for a set of three stochastic partial differential equations in one space dimension, motivated by the intermittent destabilization of tall thin convection cells by horizontal shear. Time-series are predictable in the sense that they follow limit cycles with a small variation in amplitude from cycle to cycle. Closer inspection reveals that the amplitude is determined by very small amounts of noise. (1)1999 Elsevier Science B.V. All rights reserved.
\end{abstract}

Keywords: Stochastic PDEs; Nonequilibrium dynamics; Bifurcations; Noisy periodicity; Shear instability

\section{Introduction}

Suppose a collective variable extracted from a system obeying a stochastic partial differential equation shows behavior that is 'noisily periodic': almost periodic with a small random difference in amplitude from cycle to cycle. One would expect to proceed by looking for a Hopf bifurcation in a deterministic model, then adding noise to produce a little variability. That is, one would expect the predictable part of the behavior to be deterministic and the slight variability to be stochastic. We report on a system where the opposite is true, i.e., the mean form of the time-dependent solutions is a function of the noise level, even when the latter is very small; the amplitude of the variability from cycle to cycle is independent of the noise level. The phenomenon, called noise-controlled dynamics, has been analysed in low-order models. Here we analyse a set of nonlinear stochastic partial differential equations. The particular example we shall chose is a set of model equations for the dynamics of convection cells subject to shear, obtained as a truncation of the Boussinesq equations [1-4].

In noise-controlled dynamics [5-12], timescales and qualitative behaviors of solutions are controlled by tiny amounts of additive noise. The phenomenon has been reported in a one-dimensional map [5], in repeated passage through a saddle-node bifurcation [13], and in ordinary differential equations describing the resonant interaction of wave modes [6,10], the intermittent destabilization of convection by shear [7,11], pulsating laser oscillations [9]

\footnotetext{
* Corresponding author. Fax: +1-505-6652659

E-mail address: grant@lanl.gov (G.D. Lythe) 
and plane Poiseuille flow [14]. Numerical evidence for similar behavior has been found in a set of partial differential equations describing the shear instability of thermohaline convection [8]. Recent experimental results exhibit the phenomenon in a periodically modulated laser: a change of $\mathrm{O}(1)$ in the area of the hysteresis loop is produced by tiny amounts of noise [12].

Although the phenomenon of noise-controlled dynamics is counterintuitive, its dynamics is predictable in the following sense. Collective variables perform loops whose amplitude varies from cycle to cycle. For sufficiently large noise, the simplest dynamics is found: successive maxima are drawn independently from a well-defined distribution. We shall derive an explicit formula for this distribution. For smaller values of the noise level the dynamics passes through a sequence of bifurcations that, as in many deterministic systems, can be understood from an approximate one-dimensional map.

Time-series of systems undergoing noise-controlled dynamics exhibit the following characteristic: trajectories spend most of their time in a 'slow phase', close to an invariant manifold, when variables measuring distance perpendicular to the manifold are 'slaved'. Slow phases are occasionally interrupted by short-lived 'revolts' of the slave variables (fast phases) [15]. In the analysis of noise-controlled dynamics of this type, the most important parameter is $\mu|\log \epsilon|$, where $\epsilon$ is the noise level and $1 / \mu$ is the (slow) timescale for the dynamics near the invariant manifold. The scaling of the parameters is:

$$
\epsilon \ll \sqrt{\mu} \ll 1 .
$$

In the spatially-extended system considered here, we show that the dynamics can be described in terms of the parameter

$$
\alpha=\frac{L^{2}}{4 \pi^{2}} \mu|\log \epsilon|
$$

where $L$ is the length of the spatial domain, $\mu$ is proportional to the difference between the Rayleigh number and its critical value, and $\epsilon$ is the noise magnitude.

The dynamics of a slow phase is analogous to that of a slow passage through a symmetry-breaking bifurcation. There, time-dependence of the critical parameter produces a delay that is sensitive to noise. The characteristic delay in such a dynamic bifurcation is $\sqrt{2 \mu|\log \epsilon|}$, where $\mu$ is the rate of change of the parameter [10,16-19]. Studies of dynamic bifurcations in spatially extended systems revealed, in addition, a characteristic length scale controlled by $\mu$ and $\log \epsilon[20-22]$

\section{Model equations: shear instability of convection}

We first briefly outline the motivation for the model. The vertical velocity of a fluid in cellular convective motion is a function of position that can be written

$$
\omega=A \sin \frac{2 \pi}{L} z \cos 2 \pi x,
$$

where $x$ and $z$ are the scaled horizontal and vertical directions and $L$ is the ratio of the height of a cell to its width. The presence of shear means that there is a non-zero net horizontal velocity $u$ at some values of $z$; it can be modeled by introducing the horizontal average of $u$ as a field $C(z)$ and letting the amplitude in Eq. (3) also depend on $z$. Now in an expansion in $1 / L$ (tall thin rolls) assuming a small ratio of viscous to thermal diffusivity, the leading order nonlinear interaction of $A(z)$ and $C(z)$ produces another contribution to the vertical velocity, $B(z)$, that gives the rolls a tilted appearance [3]. To solve the dynamical problem with noise, we write the three fields as stochastic 


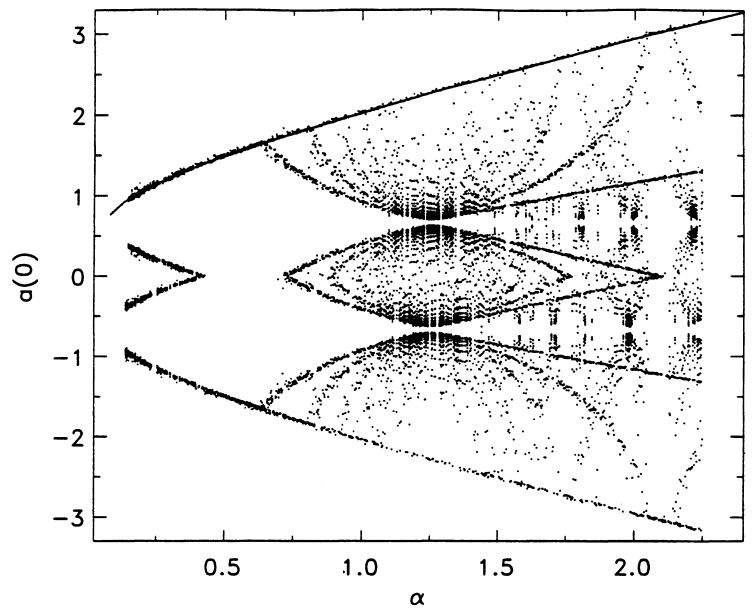

Fig. 1. Numerical bifurcation diagram. The noise level, $\epsilon$, decreases from left to right. Each dot represents a turning point of the spatial average of $A$. The parameters $L=3 \pi$ and $\mu=0.01$ are fixed. The solid line, coincident with the upper envelope of the dots, is Eq. (18), the analytically calculated maximum value of $\boldsymbol{a}_{t}(0)$.

processes depending on space and time, satisfying the following set of three stochastic partial differential equations (SPDEs):

$$
\begin{aligned}
& \partial_{t} A_{t}(z)=\mu A_{t}(z)+\partial_{z}^{2} A_{t}(z)-C_{t}(z) B_{t}(z), \quad \partial_{t} B_{t}(z)=\partial_{z}^{2} B_{t}(z)+C_{t}(z) A_{t}(z)+\epsilon \eta_{B}(z, t), \\
& \partial_{t} C_{t}(z)=\partial_{z}^{2} C_{t}(z)-A_{t}(z) \partial_{z}^{2} B_{t}(z)+\epsilon \eta_{C}(z, t) .
\end{aligned}
$$

The two space-time white noises satisfy

$$
\begin{aligned}
& \left\langle\eta_{B}(z, t) \eta_{B}\left(z^{\prime}, t^{\prime}\right)\right\rangle=\delta\left(z-z^{\prime}\right) \delta\left(t-t^{\prime}\right), \quad\left\langle\eta_{C}(z, t) \eta_{C}\left(z^{\prime}, t^{\prime}\right)\right\rangle=\delta\left(z-z^{\prime}\right) \delta\left(t-t^{\prime}\right) \\
& \left\langle\eta_{B}(z, t) \eta_{C}\left(z^{\prime}, t^{\prime}\right)\right\rangle=0 .
\end{aligned}
$$

Angled brackets denote ensemble means. These noises, and the partial derivatives, denoted by $\partial_{t}$ and $\partial_{z}^{2}$, exist in the sense of generalized functions.

The parameter $\mu$ in Eq. (4) is the scaled difference between the Reyleigh number $R$ (proportional to the temperature difference between the top and the bottom of the fluid) and the threshold for the onset of convection. The physical origin of the fields $B(z)$ and $C(z)$ requires that they be zero at $z=0$ and $z=L$; the latter is one of the boundary conditions we have used for Eq. (4). We find that different boundary conditions alter our conclusions quantitatively, but not qualitatively. In what follows, we therefore restrict ourselves to periodic boundaries, plus the conditions $\int_{0}^{L} B(z) \mathrm{d} z=0$ and $\int_{0}^{L} C(z) \mathrm{d} z=0$. A finite difference algorithm [20] with second-order time-stepping [16] was used to generate numerical solutions of the SPDEs (4).

The characteristics of the noise-controlled dynamics of this system are summarized in Fig. 1. The solutions consist of alternating slow and fast phases, and dynamics are simplest at relatively high noise levels. For long periods $\left|A_{t}(z)\right|$ is spatially uniform and slowly growing, while $B_{t}(z)$ and $C_{t}(z)$ are everywhere small. This corresponds to convection with growing amplitude. After a slow phase, there follows a short fast phase when $A_{t}(z)$ is not spatially homogeneous and $B_{t}(z)$ and $C_{t}(z)$ are of the same order of magnitude as $A_{t}(z)$. The latter phase corresponds to temporary destruction of the convection pattern by shear. Fig. 1 is constructed as follows. At each timestep, we calculate the spatial average of $A$ : 

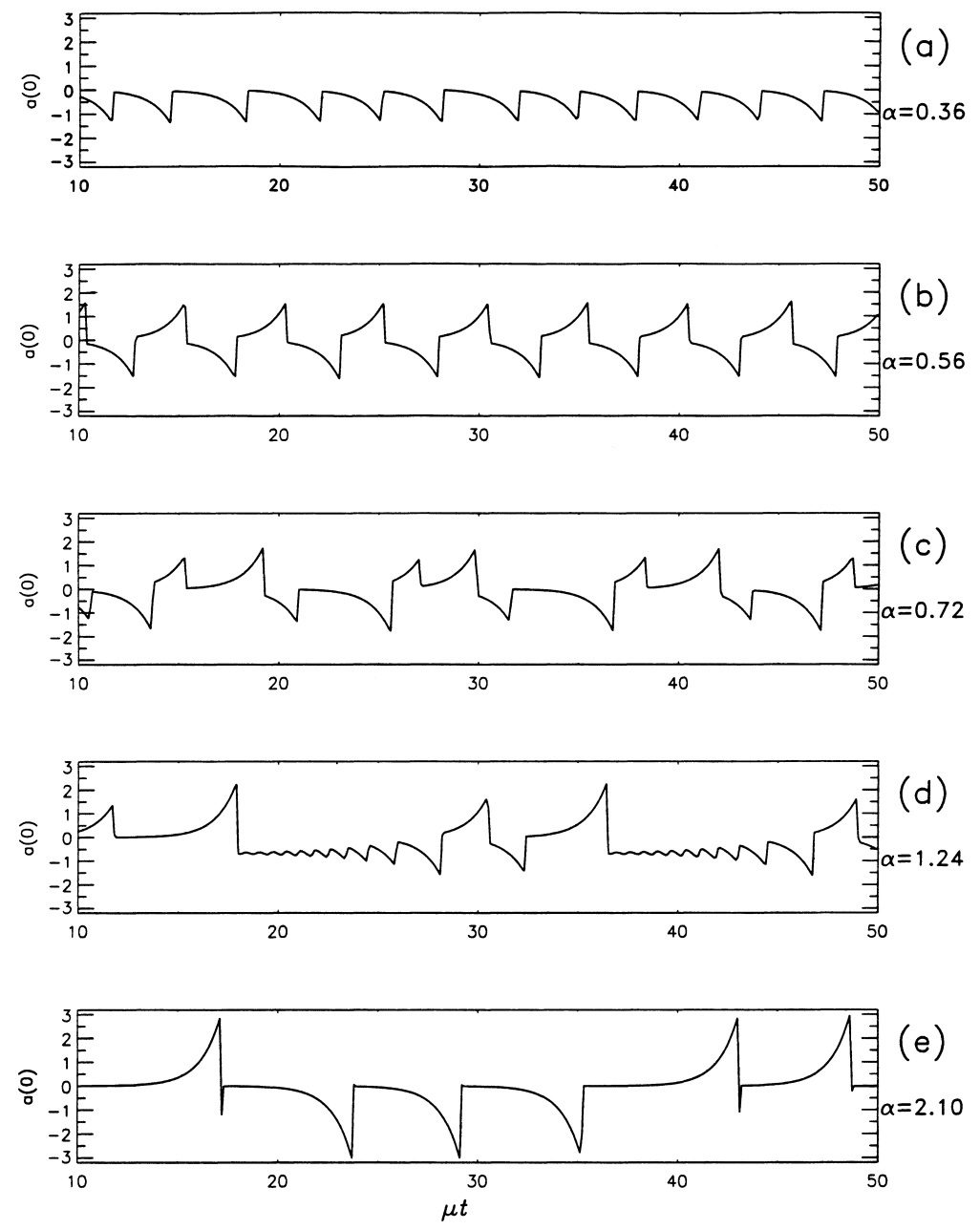

Fig. 2. Trajectories of $\boldsymbol{a}_{t}(0)$. Each graph is a time-series for the spatial average of $A$. The noise level is highest in (a) and lowest in (e); the parameters $L=3 \pi$ and $\mu=0.01$ are fixed. The time axis is $\mu t$.

$$
\boldsymbol{a}_{t}(0)=\frac{1}{L} \int_{0}^{L} A_{t}(z) \mathrm{d} z,
$$

follow its evolution and record its turning points. Each dot in Fig. 1 is a value of $\boldsymbol{a}_{t}(0)$ at a time when $\dot{\boldsymbol{a}}_{t}(0)=0$. Random initial conditions are used, then turning points are recorded after a time $\mathrm{O}\left(\mu^{-1}\right)$. The parameter $\alpha$ is defined in Eq. (2). The larger $\alpha$ is, the more complicated the solutions are (Fig. 1). Note that $\alpha$ can be increased by increasing the length of the domain $L$ or by decreasing the noise level $\epsilon$.

Fig. 2 shows the time trace of $\boldsymbol{a}_{t}(0)$ for several values of $\alpha$. The simplest case (largest noise level) is displayed in Fig. 2(a): $\boldsymbol{a}_{t}(0)$ does not change sign. (There are two distinct orbits of this type; one or the other is found at fixed $\alpha$, depending on the initial conditions.) In Fig. 2(b), alternate maxima of $\boldsymbol{a}_{t}(0)$ have opposite signs. Fig. 2(c) shows a the time-series after a period-doubling bifurcation. In Fig. 2(d) the behavior is chaotic; Fig. 2(e) is taken in a noisily periodic window.

Before proceeding to a quantitative description of the dynamics, we describe in more detail the relatively simple dynamics found at small $\alpha$. Let the Fourier coefficients $\boldsymbol{b}_{t}(k)$ and $\boldsymbol{c}_{t}(k)$ be defined as follows: 

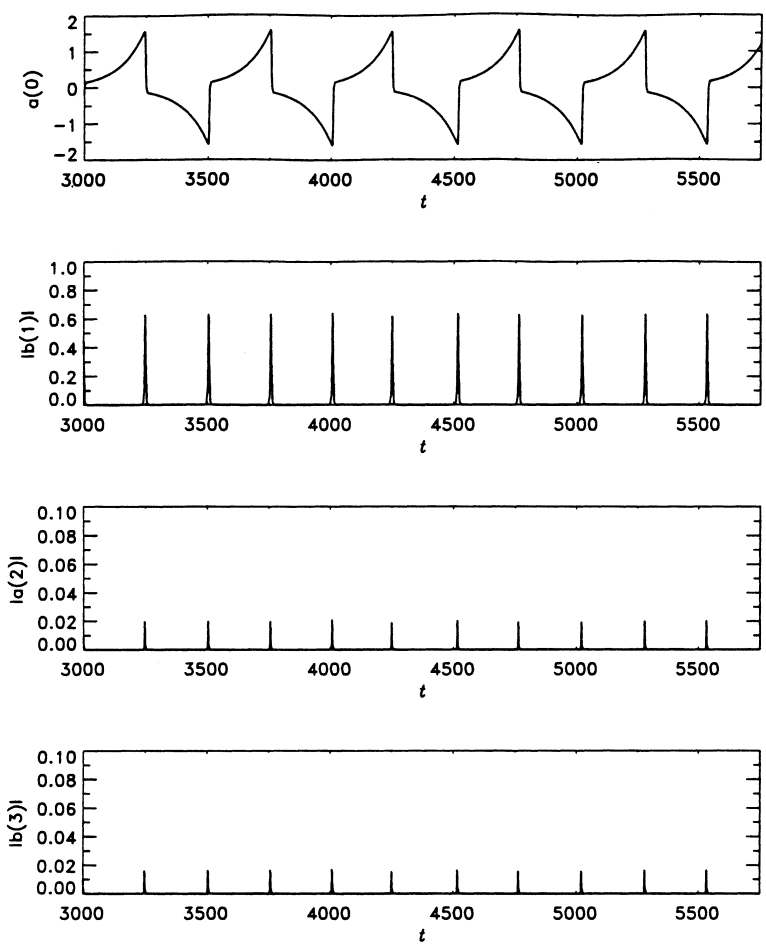

Fig. 3. Evolution of Fourier modes. The top graph is the spatial average of $A_{t}(z)$. During the long slow phases it is the only mode with large amplitude. A spike in the $k=1$ Fourier modes of $B_{t}(z)$ and $C_{t}(z)$ brings a slow phase to an end and inaugurates a short fast phase when other modes come into play. The values of the parameters are $L=3 \pi, \mu=0.01$ and $\epsilon=10^{-11}(\alpha=0.57)$.

$$
B_{t}(z)=\frac{1}{\sqrt{L}} \sum_{k=1}^{\infty} \boldsymbol{b}_{t}(k) \exp \left(\mathrm{i} k \frac{2 \pi}{L} z\right)+\text { c.c., } \quad C_{t}(z)=\frac{1}{\sqrt{L}} \sum_{k=1}^{\infty} \boldsymbol{c}_{t}(k) \exp \left(\mathrm{i} k \frac{2 \pi}{L} z\right)+\text { c.c. }
$$

Each $\boldsymbol{b}_{t}(k)$ and $\boldsymbol{c}_{t}(k)$ is a stochastic processes taking complex values. Fig. 3 shows the time trace of some of these modes for a solution of the type shown in Fig. 2(b). For long periods, $\boldsymbol{a}_{t}(0)$ is the only mode with $\mathrm{O}(1)$ amplitude. The slow growth in $\left|\boldsymbol{a}_{t}(0)\right|$ eventually produces a sudden increase in the amplitudes of $\boldsymbol{b}_{t}(1)$ and $\boldsymbol{c}_{t}(1)$, initiating a fast phase. During a fast phase, nonlinear feedback makes $\boldsymbol{A}_{t}(z)$ inhomogeneous $\left(\boldsymbol{a}_{t}(2) \neq 0\right)$ and brings higher modes into play, but $\boldsymbol{a}_{t}(0)$ is reduced very quickly and the slow phase is restored.

\section{Calculation of density of maxima}

The orbits in Figs. 2(a,b) will be described by calculating the density of the maxima of $\left|\boldsymbol{a}_{t}(0)\right|$. These maxima mark the boundary between a slow phase and the following fast phase. Successive maxima are independent, depending only the realization of the noise in the slow phase leading up to it. This is the key to the simplification of the dynamics produced by noise $[6,7,10,11]$. We shall calculate the density of maxima of $\left|\boldsymbol{a}_{t}(0)\right|$ as follows. The SPDEs are reduced to a set of stochastic ordinary differential equations (ODEs) by taking a Fourier transform in space. Using an approximation valid in the slow phase, we derive quantitatively accurate expressions for the statistics of the noise-controlled orbits for small $\alpha$, and follow the bifurcations leading to incoherence in space and time as $\alpha$ is increased. 
In the slow phase, when $\boldsymbol{a}_{t}(0)$ is approximately spatially uniform, we can set $A_{t}(z)=\boldsymbol{a}_{t}(0)$. Then the set of SPDEs (4) reduces to the following set of stochastic ODEs [16,23] for the real quantity $\boldsymbol{a}_{t}(0)$ and the complex processes $\boldsymbol{b}_{t}(k), \boldsymbol{c}_{t} k$ :

$$
\begin{aligned}
\mathrm{d} \boldsymbol{a}_{t}(0) & =\mu \boldsymbol{a}_{t}(0) \mathrm{d} t-\left(\frac{1}{L} \sum_{k=1}^{\infty}\left(\boldsymbol{b}_{t}(k)^{*} \boldsymbol{c}_{t}(k)+\boldsymbol{c}_{t}(k)^{*} \boldsymbol{b}_{t}(k)\right)\right) \mathrm{d} t, \\
\mathrm{~d} \boldsymbol{b}_{t}(k) & =\left(-\kappa^{2} \boldsymbol{b}_{t}(k)+\boldsymbol{a}_{t}(0) \boldsymbol{c}_{t}(k)\right) \mathrm{d} t+\frac{1}{\sqrt{2}} \epsilon \mathrm{d} \boldsymbol{w}_{t}(k), \\
\mathrm{d} \boldsymbol{c}_{t}(k) & =\left(-\kappa^{2} \boldsymbol{c}_{t}(k)+\kappa^{2} \boldsymbol{a}_{t}(0) \boldsymbol{b}_{t}(k)\right) \mathrm{d} t+\frac{1}{\sqrt{2}} \epsilon \mathrm{d} \boldsymbol{v}_{t}(k),
\end{aligned}
$$

where

$$
\left\langle\mathrm{d} \boldsymbol{w}_{t}(k)^{*} \mathrm{~d} \boldsymbol{w}_{t^{\prime}}\left(k^{\prime}\right)\right\rangle=\left\langle\mathrm{d} \boldsymbol{v}_{t}(k)^{*} \mathrm{~d} \boldsymbol{v}_{t^{\prime}}\left(k^{\prime}\right)\right\rangle=2 \delta\left(t-t^{\prime}\right) \delta\left(k-k^{\prime}\right) \mathrm{d} t,
$$

and

$$
\kappa^{2}=\left(\frac{2 \pi}{L} k\right)^{2} \text { for integer } k .
$$

We make the substitutions

$$
\begin{aligned}
\boldsymbol{y}_{k} & =\frac{1}{\sqrt{2 L}}\left(\frac{\boldsymbol{c}_{t}(k)}{\sqrt{\kappa}}+\boldsymbol{b}_{t}(k) \sqrt{\kappa}\right), \quad \boldsymbol{z}_{t}(k)=\frac{1}{\sqrt{2 L}}\left(\frac{\boldsymbol{c}_{t}(k)}{\sqrt{\kappa}}-\boldsymbol{b}_{t}(k) \sqrt{\kappa}\right), \\
\tilde{\epsilon} & =\epsilon \frac{1}{2} \sqrt{\kappa+\frac{1}{\kappa}} L^{-1 / 2}
\end{aligned}
$$

to convert Eq. (8) to the more convenient set of equations

$$
\begin{aligned}
\mathrm{d} \boldsymbol{a}_{t}(0) & =\mu \boldsymbol{a}_{t}(0) \mathrm{d} t-\left(\sum_{k=1}^{\infty}\left(\boldsymbol{y}_{t}(k)^{*} \boldsymbol{y}_{t}(k)-z_{t}(k)^{*} z_{t}(k)\right)\right) \mathrm{d} t, \quad \mathrm{~d} \boldsymbol{y}_{t}(k)=\kappa\left(-\kappa+\boldsymbol{a}_{t}(0)\right) \boldsymbol{y}_{t}(k) \mathrm{d} t+\tilde{\epsilon} \mathrm{d} \tilde{\boldsymbol{w}}_{t}(k), \\
\mathrm{d} \boldsymbol{z}_{t}(k) & =-\kappa\left(\kappa+\boldsymbol{a}_{t}(0)\right) z_{t}(k) \mathrm{d} t+\tilde{\epsilon} \mathrm{d} \tilde{\boldsymbol{v}}_{t}(k),
\end{aligned}
$$

where

$$
\left\langle\mathrm{d} \tilde{\boldsymbol{w}}_{t}(k)^{*} \mathrm{~d} \tilde{\boldsymbol{w}}_{t^{\prime}}\left(k^{\prime}\right)\right\rangle=\left\langle\mathrm{d} \tilde{\boldsymbol{v}}_{t}(k)^{*} \mathrm{~d} \tilde{\boldsymbol{v}}_{t^{\prime}}\left(k^{\prime}\right)\right\rangle=2 \delta\left(t-t^{\prime}\right) \delta\left(k-k^{\prime}\right) \mathrm{d} t .
$$

If only a finite number of modes $k$ is considered, Eqs. (12) are in the form studied in previous work on noise-controlled dynamics [10,11]. The analysis proceeds as follows. First, exploiting the symmetry of (12) under

$$
\boldsymbol{a}_{t}(0) \rightarrow-\boldsymbol{a}_{t}(0), \quad \boldsymbol{y}_{t}(k) \leftrightarrow \boldsymbol{z}_{t}(k),
$$

we restrict ourselves to slow phases with $\boldsymbol{a}_{t}(0)>0$. (Note that $\left|\boldsymbol{y}_{t}(k)\right|$ grows exponentially for $\left.\boldsymbol{a}_{t}(0)>\kappa\right)$. Since $\boldsymbol{y}_{t}(k)=\mathrm{O}(\epsilon)$ when $\left|\boldsymbol{a}_{t}(0)\right|$ passes through $\kappa$ from below, we can set $\dot{\boldsymbol{a}}_{t}(0)=\boldsymbol{\mu} \boldsymbol{a}_{t}(0)$ and the real and imaginary components of $\boldsymbol{y}_{t}(k)$ are Gaussian random variables with mean zero. For each $k$, the value of $\left\langle\boldsymbol{y}_{t}(k)^{*} \boldsymbol{y}_{t}(k)\right\rangle$ can be calculated analytically as a function of $\boldsymbol{a}_{t}(0)$ [10]. The important part of the evolution is for $A_{t}(z)>\kappa$, when $\left\langle\boldsymbol{y}_{t}(k)^{*} \boldsymbol{y}_{t}(k)\right\rangle$ grows exponentially fast:

$$
\left\langle\boldsymbol{y}_{t}(k)^{*} \boldsymbol{y}_{t}(k)\right\rangle \rightarrow 2 \tilde{\epsilon}^{2} \frac{1}{\kappa} \sqrt{\frac{\pi}{\mu}} \exp \left(\frac{2}{\mu} \kappa^{2} F\left(\frac{\boldsymbol{a}_{t}(0)}{\kappa}\right)\right),
$$


where

$$
F(x)=x-1-\log x .
$$

A spike in the time-series of $\left|\boldsymbol{b}_{t}(k)\right|$ appears when the exponential grows sufficiently large. The first mode to grow has $k=1$.

We denote the maximum value of $\left|\boldsymbol{a}_{t}(0)\right|$ in a cycle by $\boldsymbol{a}_{\max }$. Thus, $\boldsymbol{a}_{\max }$ is a random variable, whose distribution is as follows. The mode $k=1$ dominates the sum in Eq. (12), so $\boldsymbol{a}_{t}(0)=\boldsymbol{a}_{\max }$ when $\boldsymbol{y}_{t}(0)^{*} \boldsymbol{y}_{t}(0)=\mu \boldsymbol{a}_{t}(0)$. The maximum of the density of $\boldsymbol{a}_{\max }$ is at $\hat{a}-\Delta a$ where $\hat{a}$ satisfies $[10,11]$

$$
\kappa^{2} F\left(\frac{\hat{a}}{\kappa}\right)=\mu|\log \epsilon|+\frac{\mu}{4} \log \frac{\mu}{\pi}+\frac{1}{2} \mu \log 2 L \mu \hat{a}-\frac{1}{2} \mu \log \left(1+\frac{1}{\kappa^{2}}\right),
$$

and

$$
\Delta a=\frac{1}{2} \mu \frac{\hat{a}}{\kappa(\hat{a}-\kappa)} .
$$

We can more explicitly write the maximum of the density as a function of $\alpha$. With $k=1$ and $\mu \ll 1$, Eq. (16) reduces to

$$
\frac{\hat{a} L}{2 \pi}-\log \left(\frac{\hat{a} L}{2 \pi}\right)=1+\alpha+\mathrm{O}(\mu) .
$$

The width of the distribution is proportional to $\mu$ :

$$
\left\langle\boldsymbol{a}_{\max }^{2}\right\rangle-\left\langle\boldsymbol{a}_{\max }\right\rangle^{2}=\frac{\pi^{2}}{6} \Delta a^{2} .
$$

We can explicitly calculate the distribution of $\boldsymbol{a}_{\max }$. Using Eq. (14), we know the probability, as a function of $A_{t}(z)$ that $\boldsymbol{y}_{t}(0)^{*} \boldsymbol{y}_{t}(0)>\mu \boldsymbol{a}_{t}(0)$. The density of $\boldsymbol{a}_{\max }$ is the derivative with respect to $A_{t}(z)$ of this probability. Explicitly, the density of $\boldsymbol{u}$ defined by

$$
\boldsymbol{u}=\frac{\left(\hat{a}-\boldsymbol{a}_{\max }\right)}{\Delta a}
$$

is

$$
R_{\boldsymbol{u}}(v)=\frac{\mathrm{d}}{\mathrm{d} v} \mathcal{P}[\boldsymbol{u}<v]=\mathrm{e}^{v} \mathrm{e}^{-\mathrm{e}^{v}} .
$$

Using Eqs. (16)-(21) gives a quantitatively accurate expression for the distribution of $\boldsymbol{a}_{\max }$ (Fig. 4). This remains true when $\alpha$ is sufficiently small that the dynamics of the fast phase serves only to provide initial conditions for the slow phase.

Fig. 2 illustrates the increasingly complicated behavior of solutions as a function of $\alpha$. A description in terms of a map of successive turning points of $\boldsymbol{a}_{t}(0)$ can be constructed, where the fast phase is also analysed $[6,7,10,11]$. In the simplest approximation [6,7,10,11], the fast phase begins at $\boldsymbol{a}_{\max }$, the maximum of $\boldsymbol{a}_{t}(0)$, and ends at $\boldsymbol{a}_{t}(0)=\boldsymbol{a}_{\min }$ where $\boldsymbol{a}_{\max }+\boldsymbol{a}_{\min }=\kappa$. Using this approximation and Eq. (18), the first qualitative change in the dynamics as a function of $\alpha$, to trajectories where alternate turning points $\boldsymbol{a}_{t}(0)$ have opposite signs, is estimated to occur at

$$
\alpha=1-\log 2+\mathrm{O}(\mu) .
$$

The value of $\alpha$ for the 'period-doubling' transition from Fig. 2(b) to Fig. 2(c) can be estimated similarly. In this case the bifurcation happens when $\boldsymbol{a}_{\max }$ is large enough that the subsequent slow phase begins close to $\boldsymbol{a}_{t}(0)=\kappa$ 


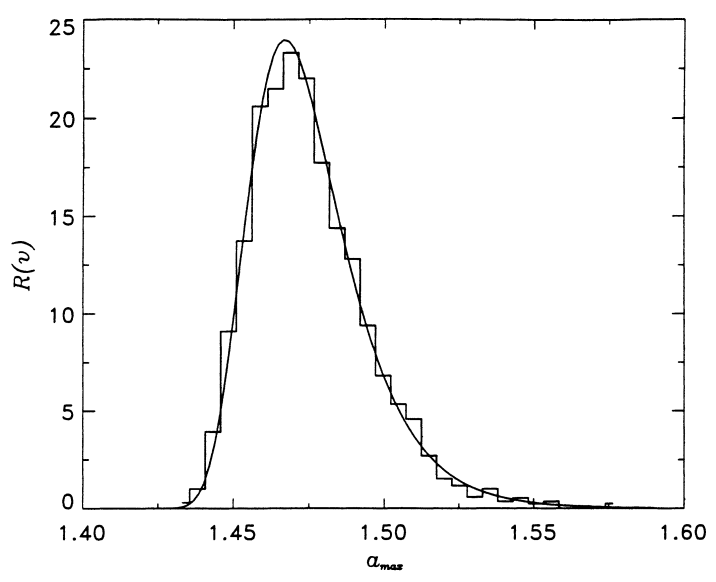

Fig. 4. Probability distribution of maxima of $\boldsymbol{a}_{t}(0)$. The histogram of turning points obtained from numerical simulation of the SPDEs is compared with the calculated density (21) (smooth line). The parameters are $L=2 \pi, \mu=0.01, \epsilon=10^{-5}(\alpha=0.115)$.

and is no longer independent of its initial conditions. More accurate approximations can be obtained by relaxing the assumption that $A_{t}(z)$ is spatially uniform and by taking into account the possibility that dominant mode among the pairs $\left(\boldsymbol{y}_{t}(k), \boldsymbol{z}_{t}(k)\right)$ may have $k>1$; one obtains a hierarchy of low-order models for convection subject to shear. The effect of a magnetic field can also be included [4].

For $\alpha>1$, solutions of Eq. (4) display noisily-periodic behavior in some windows (Fig. 2(e)), but the typical picture is one of spatial inhomogeneity with only rare coherent slow phases. The expression (18) gives a maximum possible value of $\boldsymbol{a}_{t}(0)$ that corresponds to the envelope of the points in Fig. 1, but the spatial coherence required to attain this value becomes rarer as $\alpha$ is increased. The limiting behavior for vanishingly small noise is extremely irregular.

\section{Concluding remarks}

Any differential equation modeling a physical system ignores some influences. Normally one considers the model well chosen if the ignored influences are small, rapidly varying, and have mean zero. Our example is at first glance disturbing: tiny random influences can have a huge effect. However, noisy-controlled dynamics have a non-equilibrium steady state with well-defined signatures and for which precise analytical methods with predictive power exist, once noise is taken into account. One simply needs to remember that the relevant bifurcation parameter is the logarithm of the noise level and that the larger the noise, the simpler the dynamics.

\section{References}

[1] L.N. Howard, R. Krishnamurti, Large-scale flow in turbulent convection: a mathematical model, J. Fluid Mech. 170 (1986) 385-410.

[2] M.R.E. Proctor, J.Y. Holyer, Planform selection in salt fingers, J. Fluid Mech. 168 (1986) 241-253.

[3] P.C. Matthews, M.R.E. Proctor, A.M. Rucklidge, N.O. Weiss, Pulsating waves in nonlinear magnetoconvection, Phys. Lett. A 183 (1993) 69-75.

[4] A.M. Rucklidge, P.C. Matthews, Analysis of the shearing instability in nonlinear convection and magnetoconvection, Nonlinearity 9 (1996) $311-352$.

[5] K. Matsumoto, I. Tsuda, Noise-induced order, J. Stat. Phys. 31 (1983) 87-106.

[6] D.W. Hughes, M.R.E. Proctor, Chaos and the effect of noise in a model of three-wave mode coupling, Physica D 46 (1990) 163-176. 
[7] D.W. Hughes, M.R.E. Proctor, A low order model of the shear instability of convection: chaos and the effect of noise, Nonlinearity 3 (1990) $127-153$.

[8] M.R.E. Proctor, D.W. Hughes, The false Hopf bifurcation and noise sensitivity in bifurcations with symmetry, Eur. J. Mech. B/Fluids 10 (1990) 81-86.

[9] M. Georgiou, T. Erneux, Pulsating laser oscillations depend on extremely-small-amplitude noise, Phys. Rev. A 45 (1992) 6636-6642

[10] G.D. Lythe, M.R.E. Proctor, Noise and slow-fast dynamics in a three-wave resonance problem, Phys. Rev. E 47 (1993) 3122-3127.

[11] G.D. Lythe, Dynamics controlled by additive noise, Nuovo Cimento D 17 (1995) 855-861.

[12] J.C. Celet, D. Dangoisse, P. Glorieux, G. Lythe, T. Erneux, Slowly passing through resonance strongly depends on noise, Phys. Rev. Lett. 81 (1998) 975-978.

[13] D. Sigeti, W. Horsthemke, Pseudo-regular oscillations induced by external noise, J. Stat. Phys. 54 (1989) 1217-1222.

[14] K. Fujimura, R.E. Kelly, Interaction between longitudinal convection rolls and transverse waves in unstably stratified plane Poiseuille flow, Phys. Fluids 7 (1995) 25-65.

[15] M. Diener, On the perfect delay convention, or the revolt of the slaved variables, in: H. Haken (Ed.), Chaos and Order in Nature, Springer, Berlin, 1981, pp. 249-268.

[16] K. Jansons, G. Lythe, Stochastic calculus: application to dynamic bifurcations and threshold crossings, J. Stat. Phys. 90 (1997).

[17] P. Mandel, T. Erneux, Laser Lorenz equations with a time-dependent parameter, Phys. Rev. Lett. 53 (1984) 1818-1820.

[18] M.C. Torrent, M.S. Miguel, Stochastic-dynamics characterization of delayed laser threshold instability with swept control parameter, Phys. Rev. A 38 (1988) 245-251.

[19] N.G. Stocks, R. Mannella, P.V.E. McClintock, Influence of random fluctuations on delayed bifurcations, Phys. Rev. A 40 (1989) 5361-5369.

[20] G.D. Lythe, Domain formation in transitions with noise and a time-dependent bifurcation parameter, Phys. Rev. E 53 (1996) R4271-R4274

[21] P. Laguna, W.H. Zurek, Phys. Rev. Lett. 78 (1997) 2519-2522.

[22] E. Moro, G. Lythe, Phys. Rev. E 59 (1999) R1303-R1306

[23] W. Gardiner, Handbook of Stochastic Methods, Springer, Berlin, 1990. 